\title{
SPARC overexpression suppresses radiation-induced HSP27 and induces the collapse of mitochondrial $\Delta \psi$ in neuroblastoma cells
}

\author{
SMITA TANPURE $^{1}$, JERUSHA BOYINEINI $^{1}$, MANU GNANAMONY $^{1}$, \\ REUBEN ANTONY ${ }^{2}$, KAREN S. FERNÁNDEZ ${ }^{2}$, JAIME LIBES ${ }^{2}$, JULIAN LIN $^{3}$, \\ DAVID PINSON ${ }^{4}$, PUSHPA A. JOSEPH ${ }^{4}$ and CHRISTOPHER S. GONDI ${ }^{1,4,5}$ \\ Departments of ${ }^{1}$ Internal Medicine, ${ }^{2}$ Pediatrics, ${ }^{3}$ Neurosurgery, ${ }^{4}$ Pathology and ${ }^{5}$ Surgery, \\ University of Illinois College of Medicine, Peoria, IL 61605, USA
}

Received July 27, 2016; Accepted February 1, 2017

DOI: $10.3892 / \mathrm{ol} .2017 .6075$

\begin{abstract}
Neuroblastoma is the cause of $>15 \%$ of cancer-associated mortality in children in the USA. Despite aggressive treatment regimens, the long-term survival rate for these children remains at $<40 \%$. The current study demonstrates that secreted protein acidic and rich in cysteine (SPARC) suppresses radiation-induced expression of heat shock protein 27 (HSP27) in vivo and suppresses mitochondrial membrane potential $(\Delta \psi)$ in neuroblastoma cells. In the present study, the overexpression of SPARC in SK-N-BE(2) and NB1691 neuroblastoma cell lines suppresses radiation-induced G2M cell cycle arrest, proliferation, HSP27 expression (in vitro and in vivo) and induces the collapse of the mitochondrial $\Delta \psi$. Gene ontology analysis demonstrated that the overexpression of SPARC combined with irradiation, induces the expression of dissimilar molecular function genes in SK-N-BE(2) and NB1691 cells, providing evidence of a dissimilar response signaling pathway. These results demonstrate that overexpression of SPARC suppresses radiation-induced HSP27 expression in neuroblastoma cells and the combination of SPARC and radiation induces the expression of protein 21, but suppresses neuroblastoma tumor density in in vivo mouse models. SPARC also induces mitochondrial $\Delta \psi$ collapse in SK-N-BE(2) and NB1691 neuroblastoma cells.
\end{abstract}

Correspondence to: Dr Christopher S. Gondi, Department of Internal Medicine, University of Illinois College of Medicine at Peoria, 1 Illini Drive, Peoria, IL 61605, USA

E-mail: gondi@uic.edu

Abbreviations: SPARC, secreted protein acidic and rich in cysteine; HSP27, heat shock protein 27; ECM, extracellular matrix

Key words: neuroblastoma, HSP27, SPARC, mitochondria, radiation

\section{Introduction}

Neuroblastoma is the third most prevalent malignancy in children and accounts for $>10 \%$ of cancer-associated mortality in children in the USA (1). Despite aggressive therapy, the long-term survival rates for these children remains low at $40 \%$. The long-term survival rate for children with high-risk neuroblastomas also remains poor (2). The role of irradiation in the treatment of high-risk neuroblastoma has been established $(3,4)$. As with all pediatric malignancies, the aim of radiation therapy in neuroblastoma is the eradication of the tumor, whilst minimizing collateral damage to normal tissue. Secreted protein acidic and rich in cysteine (SPARC) is part of a group of non-structural components of the extracellular matrix (ECM) that modulates interactions between cells and their environment (5-7). The role of SPARC in tumorigenesis is suggested to be cell type specific due to its diverse function in the microenvironment. In certain types of cancer, including breast, pancreatic and glioblastoma, high levels of SPARC expression have been identified to associate with disease progression and poor prognosis $(8,9)$. In other types of cancer, including ovarian (10), colorectal (11) and neuroblastoma (12-14), SPARC functions as a tumor suppressor. It has been reported that there is an inverse correlation between SPARC expression and neuroblastoma progression (14), indicating that SPARC overexpression may be an effective option for the treatment of neuroblastoma. A literature review has established that tumorigenic cell lines exhibit low or undetectable levels of SPARC, whereas non-tumorigenic cells express high levels of SPARC (9). A previous study identified heat shock protein 27 (HSP27) as a mediator of SPARC activity (15). This suggests that HSP27 may be a downstream effector of SPARC-regulated cell morphology and migration. In the present study SPARC overexpression is demonstrated to suppress radiation-induced HSP27, resulting in radio-sensitization of neuroblastoma cells.

\section{Materials and methods}

Cell lines and culture conditions. The SK-N-BE(2) neuroblastoma cell line was obtained from the American Type Culture Collection (Manassas, VA, USA) and NB1691, also a 
neuroblastoma cell line, was donated by Dr Peter Houghton (St. Jude Children's Research Hospital, Memphis, TN, USA). These cells were cultured in Opti-Mem medium (Thermo Fisher Scientific, Inc., Waltham, MA, USA) supplemented with 5\% fetal bovine serum (Atlanta Biologicals, Inc., Flowery Branch, GA, USA) and $1 \%$ penicillin/streptomycin in a humidified atmosphere containing $5 \% \mathrm{CO}_{2}$ at $37^{\circ} \mathrm{C}$.

Antibodies. Antibodies were obtained from the following sources: SPARC, (cat. no., OAAN00424; Aviva Systems Biology Corporation, San Diego, CA, USA); was used at a dilution of 1:1,000 for western blot analysis; $\beta$-actin (cat. no., sc-130300; Santa Cruz Biotechnology, Inc., Dallas, TX, USA) was used at a dilution of 1:1,000 and HSP27 (cat. no., ab2790; Abcam, Cambridge, MA) was used at a dilution of 1:200 for immunohistochemistry. Secondary antibodies used were HRP-conjugated anti-rabbit at a dilution of 1:200 for immunohistochemistry and 1:2,000 for western blot analysis (cat. no., sc-2030; Santa Cruz Biotechnology, Inc., Dallas, TX, USA) and HRP-conjugated anti-mouse antibody at a dilution of 1:200 for immunohistochemistry (cat. no., 7076; Cell Signaling Technologies, Danvers, MA).

Radiation dosage. The RS2000 radiator (Rad Source Technologies, Inc., Suwanee, GA, USA) was used, which was operated at a maximum of $150 \mathrm{kV} / 50 \mathrm{~mA}$, for all radiation treatments. All cells were treated with a single (5 Gy) dose of radiation that was administered following cell transfection. To radiate subcutaneous tumors in mice, 2 doses at 2 and 3 Gy with a maximum cumulative dose of 5 Gy was administered. The mice were shielded using lead sheets, exposing only the subcutaneous tumors.

Irradiation and SPARC overexpression in vitro. SK-N-BE(2) and NB1691 cells were transfected with SPARC overexpression plasmid RC209964 (OriGene Technologies, Inc., Rockville, MD, USA) using Lipofectamine ${ }^{\circledR}$ (Thermo Fisher Scientific, Inc.) according to the manufacturer's protocol for in vitro experiments. Cells were incubated in Opti-Mem medium supplemented with $5 \% \mathrm{FBS}$ and $1 \%$ penicillin/streptomycin in a humidified atmosphere containing $5 \% \mathrm{CO}_{2}$ at $37^{\circ} \mathrm{C}$ for $24 \mathrm{~h}$. Subsequent to this incubation cells were not treated or treated with $5 \mathrm{~Gy}$ of ionizing radiation and incubated for another $24 \mathrm{~h}$ in Opti-Mem medium supplemented with 5\% FBS and 1\% penicillin/streptomycin in a humidified atmosphere containing $5 \% \mathrm{CO}_{2}$ at $37^{\circ} \mathrm{C}$.

Western blot analysis. Following SPARC overexpression and radiation treatments, cells were obtained and total protein was isolated using Mammalian Protein Extraction reagent (Thermo Fisher Scientific, Inc.). Equal quantities of protein $(10 \mu \mathrm{g} /$ lane) measured using Pierce $660 \mathrm{~nm}$ Protein Assay (cat. no., 1861426 Thermo Fisher Scientific, Inc.) were separated in reducing conditions on $10 \%$ polyacrylamide gels. Following SDS-PAGE, the proteins were transferred on to a polyvinylidene difluoride membrane (Bio-Rad Laboratories, Inc., Hercules, CA, USA). Membranes were blocked with standard TBS Tween-20 containing 5\% non-fat skimmed milk for $1 \mathrm{~h}$ at room temperature followed by immunoprobing with primary antibody for $2 \mathrm{~h}$ at room temperature in TBS Tween-20 containing $5 \%$ non-fat skimmed milk. This was followed by washing (x4 with TBS Tween-20) followed by blocking again with TBS Tween-20 containing 5\% non-fat skimmed milk for $1 \mathrm{~h}$ at room temperature. This was followed by the addition of appropriate horseradish peroxidase-labeled secondary antibody in TBS Tween-20 containing 5\% non-fat skimmed milk and incubated for $1 \mathrm{~h}$ at room temperature followed by washing (x4 with TBS Tween-20). All incubation and washings were done on a rocking platform set at 2-strokes per min. Specific protein bands were visualized using enhanced chemiluminescence detection reagents (cat. no., 32106; Thermo Fisher Scientific, Inc.).

Expression analysis of protein 21 (p21) and HSP27 using a spotted antibody array. An antibody array for the detection of HSP27 and p21 was obtained from RayBiotech Human Apoptosis array C1 (catalogue no., AAH-APO-1; RayBiotech, Inc., Norcross, GA, USA) and processed according to the manufacturer's protocol. Briefly, $80 \%$ confluent petri plates of SK-N-BE(2) and NB1691 cells were transfected with SPARC overexpression plasmid, followed by radiation treatment as previously described. Total protein was isolated using Mammalian Protein Extraction reagent (Thermo Fisher Scientific, Inc.) and equal quantities of protein $(500 \mu \mathrm{g})$ measured using Pierce 660 nm Protein Assay (cat. no., 1861426 Thermo Fisher Scientific, Inc.) were added to the provided antibody array and processed according to the manufacturer's protocol, expression of HSP27 and p21 was determined by measuring signal intensities compared to untreated controls.

Cell cycle analysis. Cell cycle distribution of SPARC overexpressing and irradiated SK-N-BE(2) cells, and NB1691 neuroblastoma cells was analyzed using flow cytometry (FACS Calibur System; BD Bioscience, San Jose, CA, USA) with excitation at a wavelength of $488 \mathrm{~nm}$ and an emission of $639 \mathrm{~nm}$, following propidium iodide staining according to standard protocols (9). A total of 10,000 cells were sorted to determine cell cycle phase using the Cell Quest Pro software version 5.2.1 (BD Bioscience, San Jose, CA, USA).

Cell proliferation assay. An MTT cell proliferation assay (cat. no., 50-213-524, Thermo Fisher Scientific, Inc.) was performed using SPARC overexpressing and irradiated SK-N-BE(2) and NB1691 neuroblastoma cells plated in 96 well plates (density, 2,000 cells/well). After $72 \mathrm{~h}$ of incubation in Opti-Mem medium supplemented with 5\% FBS and 1\% penicillin/streptomycin in a humidified atmosphere containing $5 \%$ $\mathrm{CO}_{2}$ at $37^{\circ} \mathrm{C}$, MTT was added at a concentration of $0.5 \mathrm{mg} / \mathrm{ml}$ to each well. Plates were incubated for $3 \mathrm{~h}$ at $37^{\circ} \mathrm{C}$. Following incubation, $100 \mu \mathrm{l}$ of dimethyl sulfoxide was added and the absorbance was measured at a wavelength of $550 \mathrm{~nm}$ and presented as the survival percentage.

In vitro scratch assay. The in vitro scratch assay was performed as previously described $(16,17)$. Briefly, SK-N-BE(2) and NB1691 neuroblastoma cells were plated in 24-well plates (density, 10,000 cells/well). The cells were transfected with SPARC overexpression plasmid followed by treatment with $5 \mathrm{~Gy}$ ionizing radiation after $24 \mathrm{~h}$ at $37^{\circ} \mathrm{C}$. The cell monolayer was scratched with a $100 \mu \mathrm{l}$ pipette tip and the migration into 
the gap was measured over time using the EVOS-FL cell imaging system, magnification, $\mathrm{x} 4$ and graphically presented.

Determination of mitochondrial membrane potential $(\Delta \psi)$. The mitochondrial $\Delta \psi$ was measured using the MitoProbe ${ }^{\mathrm{TM}}$ JC-1 Assay kit (cat. no., M34152, Thermo Fisher Scientific, Inc.). Briefly, SK-N-BE(2) and NB1691 neuroblastoma cells were plated in 24 well plates (density, 20,000 cells/well). The cells were transfected with SPARC overexpression plasmid followed by treatment with $5 \mathrm{~Gy}$ of ionizing radiation. A total of $48 \mathrm{~h}$ after irradiation, cells were stained with JC-1 dye at a final concentration of $2 \mu \mathrm{M}$ and the cells were incubated at $37^{\circ} \mathrm{C}, 5 \% \mathrm{CO}_{2}$, for $30 \mathrm{~min}$ and sorted using flow cytometry $(10,000$ cells $)$ and fluorescence emission at a wavelength of $\sim 590 \mathrm{~nm}$ was quantified and graphically presented. Staurosporine (cat. no., 11055682001 Roche Diagnostics Mannheim, Germany) was used as a positive control (5 $\mu \mathrm{M})$ to determine experimental validity $(18,19)$.

Caspase assay. Caspase activation was detected using the CellEvent ${ }^{\mathrm{TM}}$ Caspase-3/7 Green ReadyProbes from Molecular Probes $^{\mathrm{TM}}$ (cat. no., R3711; Thermo Fisher Scientific, Inc.), according to the manufacturer's protocol. SK-N-BE (2) and NB1691 cells were plated in chamber slides and transfected with Lipofectamine ${ }^{\circledR}$, according to standard protocols using (20) the SPARC overexpression plasmid. A total of $24 \mathrm{~h}$ after transfection, cells were exposed to $5 \mathrm{~Gy}$ of ionizing radiation and incubated for $24 \mathrm{~h}$ in Opti-Mem medium supplemented with $5 \%$ FBS and $1 \%$ penicillin/streptomycin in a humidified atmosphere containing $5 \% \mathrm{CO}_{2}$ at $37^{\circ} \mathrm{C}$. Following this $24 \mathrm{~h}$ incubation, media was removed followed by the addition of $60 \mu \mathrm{l}$ of CellEvent Caspase-3/7 Green ReadyProbe. Cells were observed using a confocal microscope (aperture setting 2). Nuclei were visualized using propidium iodide. The percent of cells exhibiting activated caspase $3 / 7$ was determined by counting the number of cells with green florescence in 10 random fields.

Radiation and SPARC overexpression in vivo. All animal studies were according to instructional approved protocols by the IRB of the University Of Illinois College Of Medicine at Peoria (Peoria, USA). Female athymic Nude mice (Foxn $1^{\text {nu }}$ ) obtained from Jackson Laboratories (Bar Harbor, ME) were subcutaneously implanted on the right rear flank with $1 \times 10^{6}$ SK-N-BE(2) or NB1691 cells harvested at the log phase $(n=5)$ using a 20 gauge needle attached to a syringe at a maximum volume of $200 \mu \mathrm{l}$ in serum free OptiMem media. The tumors were allowed to grow until $5 \mathrm{~mm}$ in diameter and followed by intratumoral injections of $100 \mu \mathrm{g}$ of SPARC overexpression plasmid solution in phosphate buffer saline $(\mathrm{NaCl} 137 \mathrm{mmol} / \mathrm{l}$, $\mathrm{KCl} 2.7 \mathrm{mmol} / \mathrm{l}, \mathrm{Na} 2 \mathrm{HPO} 410 \mathrm{mmol} / \mathrm{l}, \mathrm{KH} 2 \mathrm{PO} 41.8 \mathrm{mmol} / \mathrm{l}$ ) on day 5,15 and 20 . Controls were untreated or treated with $100 \mu \mathrm{g}$ of empty plasmid vector (pGEM) in phosphate buffer saline. Tumors were irradiated on day 7 at 2 Gy and on day 17 at $3 \mathrm{~Gy}$ each with a cumulative dose of $5 \mathrm{~Gy}$. Mice were euthanized by carbon dioxide asphyxiation when they lost $>20 \%$ of body weight or had altered behavior when ambulating, feeding or grooming. Tumors were obtained on day 30 and fixed in $10 \%$ phosphate-buffered formaldehyde for a minimum of $24 \mathrm{~h}$ at room temperature and processed for paraffin embedding using an automated tissue processor (Tissue-Tek VIP Vacuum Infiltration Processor; Sakura Finetek USA, Inc., Torrance, CA) and sectioning using a manual microtome set at $6 \mu \mathrm{m}$ (Leitz 1512 , Ramsey, MN), prior to sectioning the paraffin blocks were cooled to $-4^{\circ} \mathrm{C}$. Serial section were taken and allowed to float over warm deionized water $\left(42^{\circ} \mathrm{C}\right)$ and collected on subbed slides (21).

Immunohistochemistry. Tissue sections (thickness, 6- $\mu \mathrm{m}$ ) obtained from the paraffin blocks were. The paraffin sections were deparaffinized in $100 \%$ xylene (20 min at room temperature) followed by $100 \%$ ethanol ( $2 x, 15$ min each). The sections were then rehydrated in serial ethanol concentrations $(100,90$, $70,50,30$ and $20 \%$ ) for 10 min each. Subsequent to rehydration the sections were incubated in standard PBS for $10 \mathrm{~min}$ at room temperature followed by incubation for $30 \mathrm{sec}$ in PBS at $80^{\circ} \mathrm{C}$ for antigen retrieval. The sections were then treated with $1 \% \mathrm{H}_{2} \mathrm{O}_{2}$ at room temperature for $5 \mathrm{~min}$ to inactivate peroxidases if any. The sections were then incubated in $1 \%$ BSA solution in PBS at room temperature for $1 \mathrm{~h}$ followed by incubation with monoclonal HSP27 antibody (1:200 dilution for $2 \mathrm{~h}$ at room temperature; Santa Cruz Biotechnology, Inc.). The sections were then washed in PBS at room temperature (3x) followed by incubation with appropriate horseradish peroxidase-conjugated secondary antibody at a dilution of 1:200 in 1\% BSA in PBS for $1 \mathrm{~h}$. The slides were the washed in PBS three times (5 min each at room temperature) followed by the addition of DAB Peroxidase (HRP) Substrate (cat. no., SK-4100, Vector Labs Burlingame, CA). The DAB reaction proceeded until the desired color developed, and was stopped by washing with PBS at room temperature. Nucleus was counterstained with hematoxylin. Negative control slides were obtained by treating with non-specific Immunoglobulin G. Sections were mounted and analyzed using the EVOS-FL cell imaging system. The intensity of HSP27 expression per unit area was measured in arbitrary pixel units using Image $\mathrm{J}$ (version 1.4) and represented as raw units.

Tumor density measurement. Hematoxylin stained tumor sections were analyzed and an average number of tumor cells in random 10 different fields in an area of $100 \mu \mathrm{m}^{2}$ was measured and graphically presented (300 dpi image at magnification x10).

RNA isolation. SPARC-overexpressing NB1691 and SK-N-BE(2) cells were treated with 5 Gy of ionizing radiation as described above. Total RNA was isolated using the TRIzol method as per standard protocols (cat. no., 15596026; Thermo Fisher Scientific, Inc.) (22) and RNA quantity and quality were measured by NanoDrop ND-1000.

RNA labeling and array hybridization (Arraystar, Inc.). Sample labeling and array hybridization of total RNA isolated from SPARC-overexpressing NB1691 and SK-N-BE (2) treated with or without $5 \mathrm{~Gy}$ of ionizing radiation were performed according to the Agilent One-Color Microarray-Based Gene Expression Analysis protocol (Agilent Technologies, Inc., Santa Clara, CA, USA). The array consisted of 27,958 Entrez Gene RNAs, content sourced from RefSeq Build 36.3, Ensemble Release 52, Unigene Build 216 and GenBank 
(April 2009) on a 4x44K slide formats (http://www. agilent.com/cs/library/usermanuals/Public/G4140-90040_

GeneExpression_OneColor_6.9.pdf). Total RNA from each sample was linearly amplified and simultaneously labeled with Cy3-UTP (cat. no., NEL580; PerkinElmer, Inc., Waltham, MA, USA). The labeled cRNAs were purified by RNeasy Mini kit as per manufacturer's protocol (Qiagen, Inc., Valencia, CA, USA). A total of $1 \mu \mathrm{g}$ of each labeled cRNA was fragmented by adding $11 \mu \mathrm{l} 10 \mathrm{X}$ Blocking Agent and $2.2 \mu 1$ of 25X Fragmentation Buffer, then heated at $60^{\circ} \mathrm{C}$ for $30 \mathrm{~min}$, and finally $55 \mu \mathrm{l} 2 \mathrm{X}$ GE Hybridization buffer was added to dilute the labeled cRNA. A total of $100 \mu$ l hybridization solution was dispensed into the gasket slide and assembled to the gene expression microarray slide. The slides were incubated for $17 \mathrm{~h}$ at $65^{\circ} \mathrm{C}$ in an Agilent Hybridization Oven (Agilent Technologies, Inc.). The hybridized arrays were washed using Gene Expression wash buffer with $0.005 \%$ Triton-X102 twice in wash buffer 1 at room temperature for $1 \mathrm{~min}$ and once in wash buffer 2 at $37^{\circ} \mathrm{C}$ for 1 min followed by scanning using the Agilent DNA Microarray Scanner (part no. G2505C; Agilent Technologies, Inc.). RNA labeling, hybridization and data analysis was performed by Arraystar Inc (Arraystar, Inc., Rockville, MD, USA) using Agilent Feature Extraction software (version 11.0.1.1; Agilent Technologies, Inc.).

Gene ontology enrichment data analysis. This analysis was performed using Agilent Feature Extraction software (version 11.0.1.1; Agilent Technologies, Inc.) was used to analyze the acquired array images. Quantile normalization and subsequent data processing were performed with using the GeneSpring GX software (version 12.0; Agilent Technologies, Inc.). Following quantile normalization of the raw data, genes exhibiting enriched expression were selected for further data analysis. Differentially expressed genes with statistical significance were identified through Fold Change filtering set to 1. Gene Ontology analysis was performed using the standard enrichment computation method to functionally profile gene sets and was graphically presented using Gene Ontology Consortium tools (23) (http://geneontology.org/).

Statistical methods. All data are expressed as the mean \pm standard deviation. Statistical analysis was performed using Student's $t$-test. $\mathrm{P} \leq 0.05$ was considered to indicate a statistically significant difference. Regression analysis was used to determine the statistical validity of migration. All experiments were performed in triplicate, or as indicated. For the pathway analysis the enrichment P-value of the PathwayID used the EASE method, EASE Score is a modified Fisher's exact probability by penalizing (subtract) the count of positive agreement by 1 .

\section{Results}

Overexpression of SPARC suppresses proliferation and radiation-induced $G 2 M$ arrest. To determine whether overexpression of SPARC suppresses proliferation and radiation-induced $\mathrm{G} 2 \mathrm{M}$ arrest, SPARC was overexpressed in NB1691 and SK-N-BE(2) neuroblastoma cells (Fig. 1A). The MTT assay demonstrated that in SPARC overexpressing and irradiated NB1691 cells, the proliferation rate decreased significantly $(\mathrm{P}<0.0001)$, whereas in SK-N-BE2 cells an increase in proliferation was observed in SPARC overexpressing cells compared with the controls (Fig. 1B), notably SPARC overexpressed and radiation treated SK-N-BE2 cells showed decreased survival compared with the untreated controls (Fig. 1B), this observation may be due to the varied p53 status of NB1691 (wt) and SK-N-BE(2) (mt). Furthermore, it was observed that radiation-induced accumulation of cells in the $\mathrm{G} 2$ phase and the addition of SPARC overexpression suppressed G2 phase accumulation in NB1691 and SK-N-BE(2) (Fig. 1C). Quantitative analysis of G2M phase identified that radiation alone in NB1691 cells resulted in $34 \pm 6 \%$ cells in the $\mathrm{G} 2 \mathrm{M}$ phase $(\mathrm{P}=0.0024)$, whereas the addition of SPARC overexpression and radiation exhibited a $28 \pm 4 \%(\mathrm{P}=0.0076)$ increase in $\mathrm{G} 2 \mathrm{M}$ phase cells compared with controls of which $12 \pm 3 \%$ cells were in the G2M phase. Similar results were observed when SK-N-BE(2) cells were treated with radiation alone, which resulted in a $\mathrm{G} 2 \mathrm{M}$ phase accumulation of $39 \pm 4 \%(\mathrm{P}=0.0006)$ and the overexpression of SPARC and radiation exhibited a G2M phase accumulation of $28 \pm 5 \%$ ( $\mathrm{P}=0.0056$; Fig. 1D).

SPARC overexpression suppresses radiation-induced HSP27. To determine whether the overexpression of SPARC influences the expression of HSP27 in vivo, the nude mice subcutaneous tumor model of neuroblastoma was used in the current study (11). The results demonstrated that radiation exposure increased HSP27 expression in NB1691 and SK-N-BE(2) subcutaneous tumors. SPARC overexpression reduced the radiation-induced HSP27 expression indicating that SPARC may negatively regulate HSP27 (Fig. 2A). Quantitative analysis of HSP27 expression in the tumors identified radiation-induced HSP27 expression in $55 \pm 6 \%$ of NB1691 cells, whereas the addition of SPARC reduced the radiation-induced HSP27 expression to $12 \pm 2 \%$ of cells compared with untreated controls of which the expression rate of HSP27 was determined to be $8 \pm 2 \%$ ( $\mathrm{P}=0.0162$; Fig. $2 \mathrm{~B}$ ). In SK-N-BE(2) cell tumors that were exposed to radiation, HSP27 expression was increased to $59 \pm 2 \%$ and the addition of SPARC reduced the radiation-induced $\mathrm{HSP} 27$ expression to $16 \pm 4 \%$ $(\mathrm{P}=0.0026)$. To additionally determine whether SPARC causes a reduction in tumor burden, relative tumor density was evaluated by measuring the number of cells per unit area in a hematoxylin stained section. Untreated NB1691 tumors exhibited the highest tumor density at $138 \pm 10$ cells per $100 \mu \mathrm{m}^{2}$. The addition of radiation treatment decreased the tumor density to $112 \pm 8$ cells per $100 \mu \mathrm{m}^{2}$. Further addition of SPARC overexpression reduced the tumor density further to $68 \pm 5$ cells per $100 \mu \mathrm{m}^{2}(\mathrm{P}=0.0001$ Fig. 2B) compared with the radiated controls. Tumor density of NB1691-derived tumors following treatment with SPARC alone was similar to the densities of irradiated tumors $(110 \pm 6$ cells per $100 \mu \mathrm{m}^{2}$ ). SK-N-BE(2)-derived tumors exhibited similar tumor density patterns with the control group at $135 \pm 7$ cells per $100 \mu \mathrm{m}^{2}$, SPARC overexpressing tumors at $100 \pm 12$ cells per $100 \mu \mathrm{m}^{2}$, radiation treated tumors at $94 \pm 5$ cells per $100 \mu \mathrm{m}^{2}$ and SPARC and radiation treated tumors at $65 \pm 11$ cells per $100 \mu \mathrm{m}^{2}$ $(\mathrm{P}=0.0021$; Fig. 2B). Similarly, in vitro cells treated with radiation induced the expression of the radiation response gene HSP27, in NB1691 and SK-N-BE(2) cells. Overexpression of SPARC accompanied with irradiation suppressed HSP27 expression in NB1691 (5-fold) and SK-N-BE(2) (2-fold) cells. Furthermore, it was also observed that the expression of p21 increased in SPARC overexpressing irradiated (5 Gy) cells: 4-fold in NB1691 
A

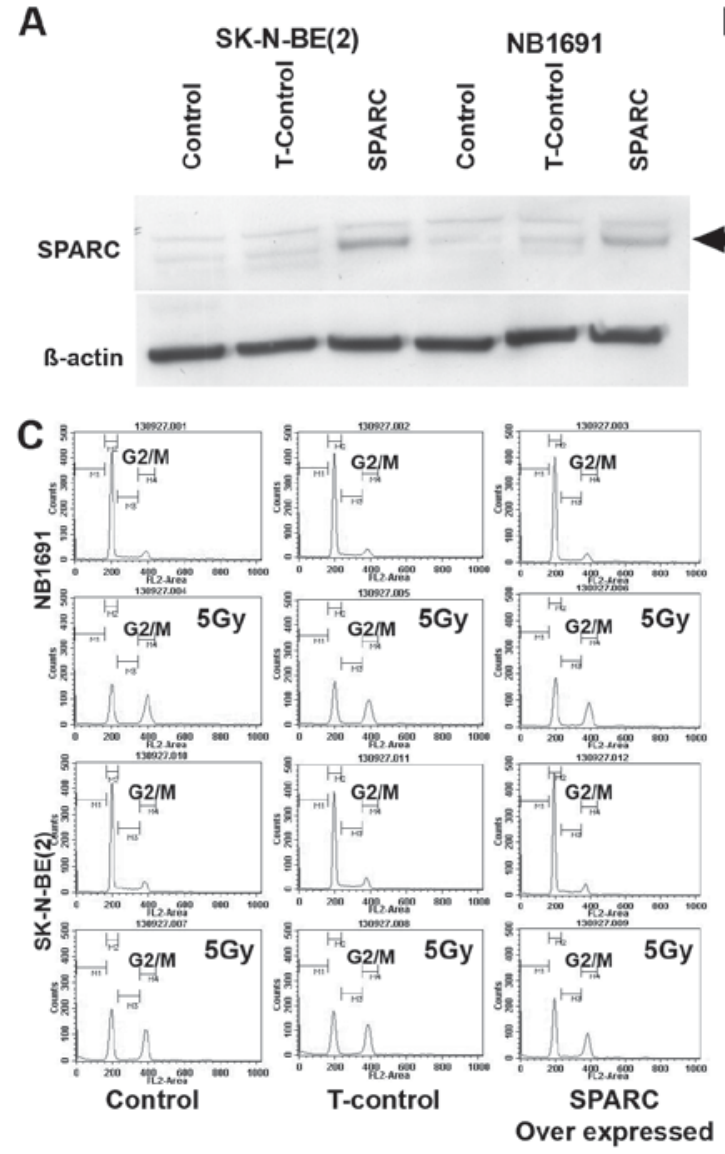

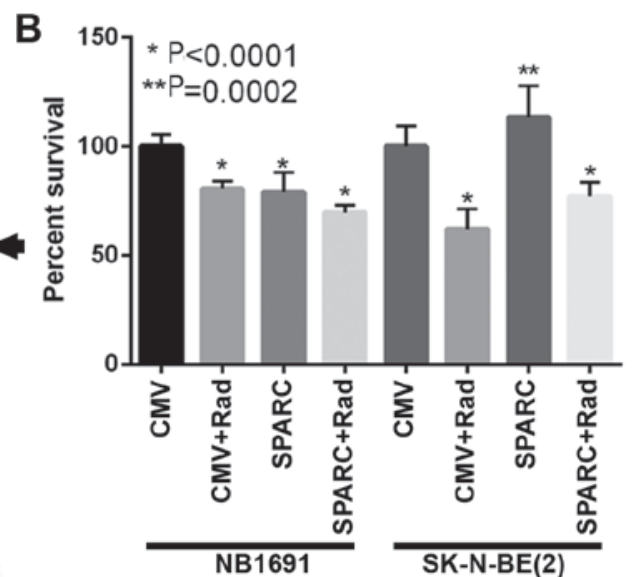

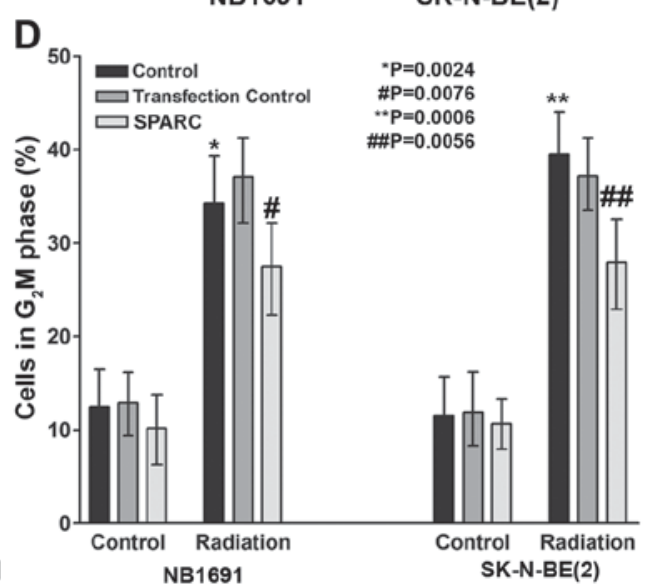

Figure 1. Overexpression of SPARC and irradiation suppresses proliferation and reduces $\mathrm{G}_{2} \mathrm{M}$ cell cycle arrest in NB1691 and SK-N-BE(2) neuroblastoma cells. (A) SPARC overexpressing and radiation-treated cells were harvested and total proteins (10 $\mu \mathrm{g} / \mathrm{lane})$ were electrophoresed under reducing conditions on $10 \%$ polyacrylamide gels and immunoprobed for SPARC expression with $\beta$-actin as a loading control. (B) Effect of SPARC overexpression and irradiation on proliferation was determined using an MTT assay and results are presented as percent survival, results were compared to untreated controls $\left({ }^{*} \mathrm{P}<0.0001\right.$, ${ }^{* *} \mathrm{P}=0.0002$ ). (C) To determine the effect of SPARC overexpression and irradiation on the cell cycle, propidium iodide stained cells were sorted using flow cytometry and (D) the percentage of cells in the G2M phase were quantified. SPARC, secreted protein acidic and rich in cysteine; HSP27, heat shock protein 27. CMV, Cells transfected with control plasmid containing CMV promoter with no SPARC insert; CMV-R, Cells transfected with control plasmid containing CMV promoter with no SPARC insert along with radiation; Control, untreated cells; T-control=transfection control/Cells transfected with control plasmid.

cells; 2.5-fold SK-N-BE(2) cells (Fig. 2C and D). There was an inverse association between the expression of HSP27 and p21, in SPARC overexpressing irradiated environment $(\mathrm{P}<0.05)$, this is in accordance with a previous study that established that HSP27 protects cells from the p21 apoptotic pathway (Fig. 2E) (12).

SPARC overexpression and irradiation suppresses tumor cell migration. To determine whether SPARC overexpression suppresses tumor cell migration, the in vitro wound healing assay (13) was performed. The overexpression of SPARC and irradiation reduced the migration potential of NB1691 and SK-N-BE(2) cells (Fig. 3A and B). Quantitative analysis demonstrated that SPARC overexpressing cells had a reduced migration potential in NB1691 $\left(300 \pm 30 \mu \mathrm{m} ; \mathrm{R}^{2}=0.77\right)$ and SK-N-BE(2) $\left(40 \pm 8 \mu \mathrm{m} ; \mathrm{R}^{2}=0.90\right)$ after 4 days of migration compared with controls (NB1691, 100 $\pm 10 \mu \mathrm{m}$; SK-N-BE(2), $3 \pm 1 \mu \mathrm{m}$; Fig. 3C and D).

SPARC overexpression and irradiation induces mitochondrial $\Delta \psi$ collapse and induces caspase mediated cell death. A previous study established that targeting HSP27 promotes radio sensitization (14). Previous studies have also demonstrated that HSP27 protects against mitochondrial-induced apoptosis $(15,16)$. Therefore the present study aimed to determine whether SPARC facilitates the suppression of HSP27-mediated protection of mitochondrial $\Delta \psi$. NB1691 and SK-N-BE(2) cells with overexpressed SPARC were irradiated and the mitochondrial $\Delta \psi$ was determined from MitoProbe ${ }^{\mathrm{TM}}$ JC-1 stained cells evaluated using flow cytometry analysis. SPARC overexpression reduced the mitochondrial $\Delta \psi$ to $70 \pm 3 \%$ and the addition of irradiation reduced the mitochondrial $\Delta \psi$ to $64 \pm 6 \%$ in NB1691 cells when compared to untreated control $(\mathrm{P}<0.05)$. In $\mathrm{SK}-\mathrm{N}-\mathrm{BE}(2)$ cells, SPARC overexpression alone reduced the mitochondrial $\Delta \psi$ to $46 \pm 3 \%$ and the addition of irradiation reduced the mitochondrial $\Delta \psi$ to $53 \pm 2 \%$ when compared with the untreated control $(\mathrm{P}<0.05)$ (Fig. 4A-C).

Overexpression of SPARC accompanied with irradiation induces differential molecular function response genes in NB1691 and SK-N-BE(2) neuroblastoma cells. To determine the molecular function response genes in NB1691 and SK-N-BE(2) cells overexpressing SPARC and treated with irradiation, genome expression analysis was performed using the Allegiant whole genome array (Arrarstar Service). SPARC overexpression accompanied with irradiation induced the 


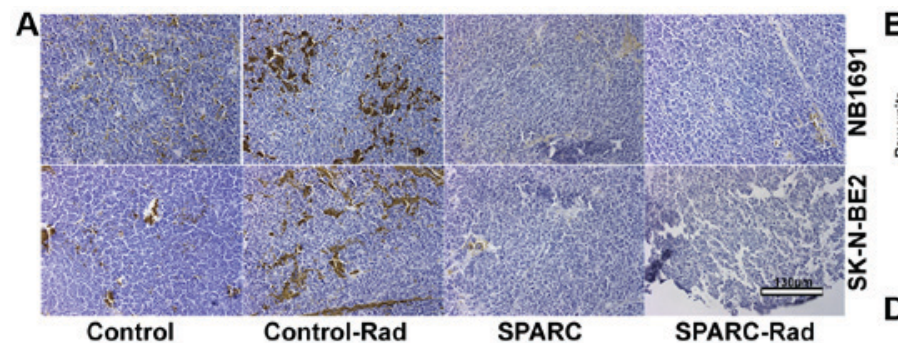

B $\quad P=0.0026$

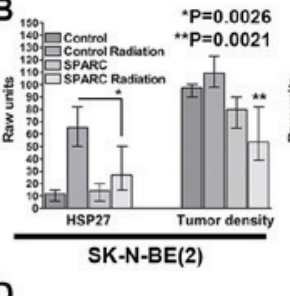

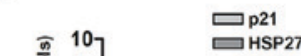

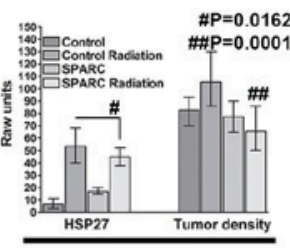

NB1691

C
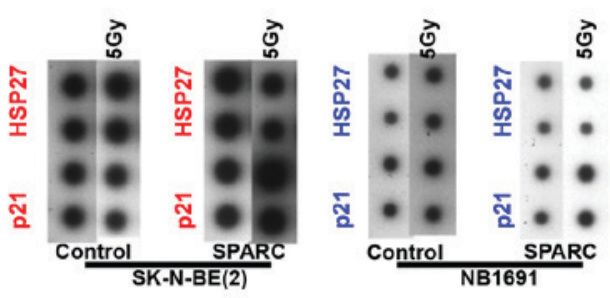

E SPARC

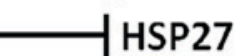

HSP27 Radiation

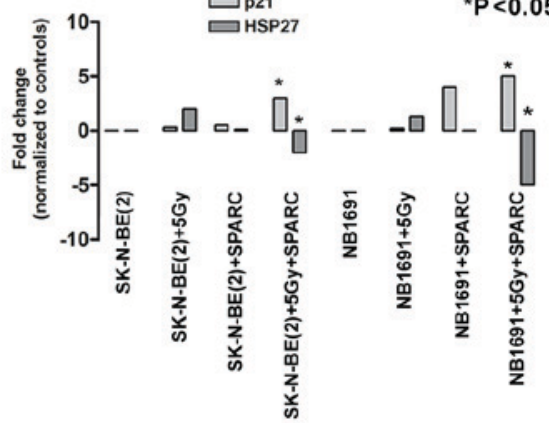

Figure 2. Overexpression of SPARC suppresses radiation-induced HSP27 expression and induces p21 expression in NB1691 and SK-N-BE(2) neuroblastoma cells. (A) To determine whether overexpressed SPARC suppressed radiation-induced HSP27 in vivo, a subcutaneous tumor model was used and expression levels of HSP27 were determined using immunohistochemistry. Brown color shows expression of HSP27; as a counterstain Hematoxylin was used to stain nuclei seen as dark blue (Scale bar $130 \mu \mathrm{m}$ ). (B) Expression levels of HSP27 and tumor density were quantified compared to untreated controls or as indicated and graphically presented. (C) To determine the relative expression levels of HSP27 and p21 in SPARC overexpressing and radiation-treated cells, an antibody array was used to determine expression of these proteins. (D) Expression of HSP27 and p21 was quantified as fold change and graphically presented (P<0.05). (E) Schematic representation of SPARC mediated radio-sensitization. SPARC, secreted protein acidic and rich in cysteine; HSP27, heat shock protein 27; p21, protein 21; Rad, radiation-treated.
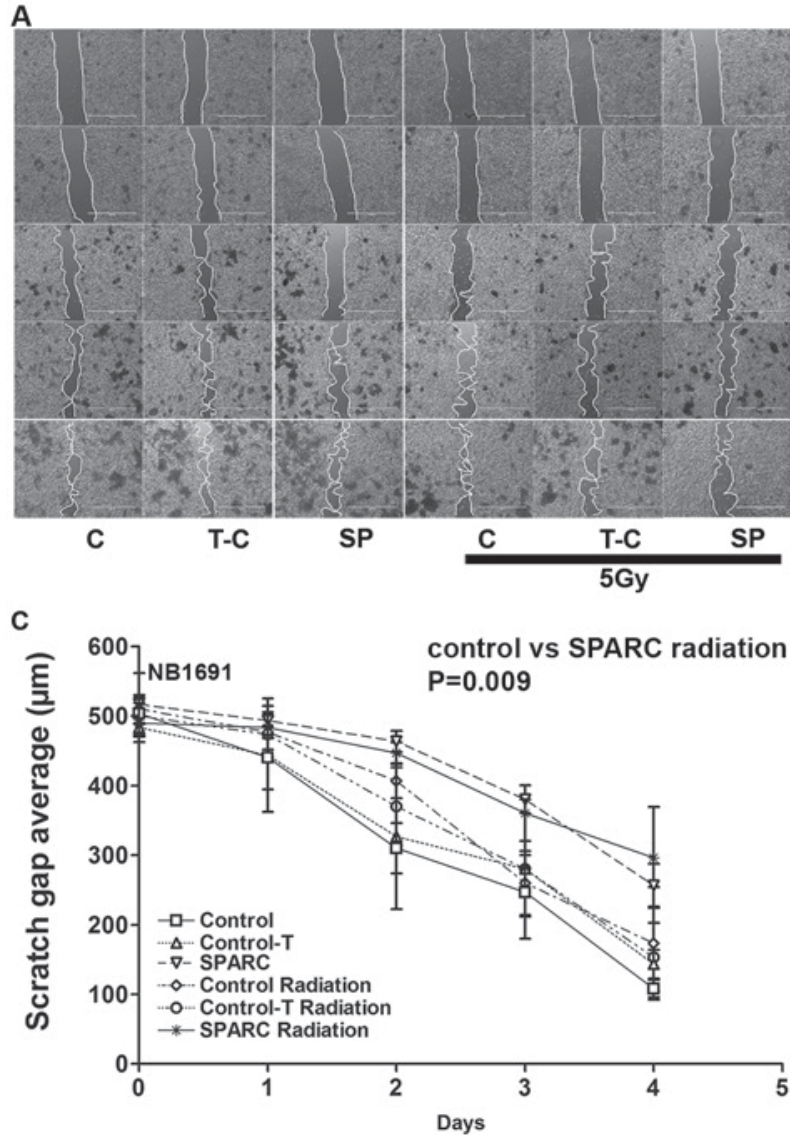
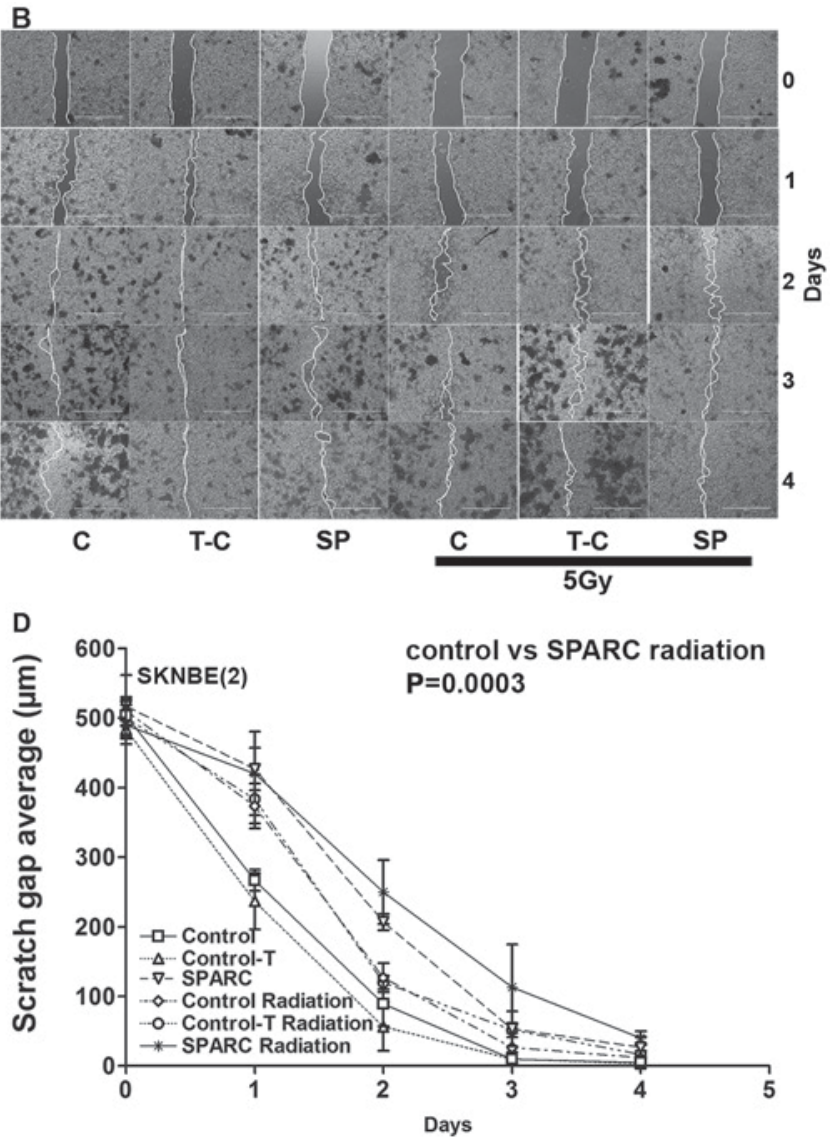

Figure 3. Overexpression of SPARC and radiation treatment suppresses migration in NB1691 and SK-N-BE(2) neuroblastoma cells. A total of 10,000 cells/well of (A) SK-N-BE(2) and (B) NB1691 neuroblastoma cells were plated in a 24-well plate. The cells were transfected with SPARC overexpressing vector and treated with $5 \mathrm{~Gy}$ ionizing radiation after $24 \mathrm{~h}$. The cell monolayer was scratched with a $100 \mu \mathrm{l}$ pipette tip and migration was measured over time and (C and D) graphically presented. SPARC, secreted protein acidic and rich in cysteine; C, control; C1, transfection control; T, control transfected vector; SP, secreted protein acidic and rich in cysteine overexpression. 

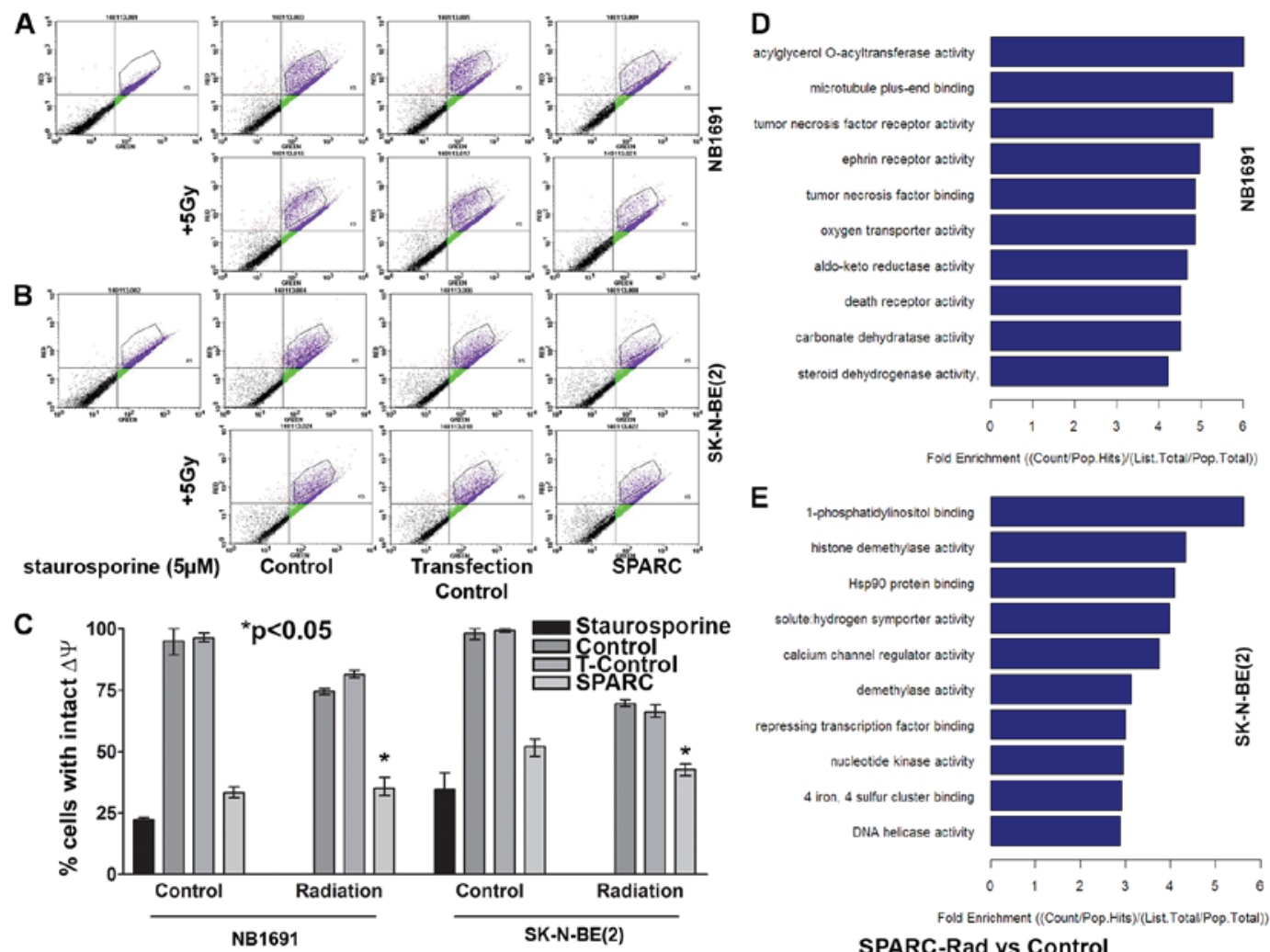

SPARC-Rad vs Control

Figure 4. Overexpression of SPARC and irradiation suppresses mitochondrial $\Delta \psi$ and induces dissimilar molecular pathways in NB1691 and SK-N-BE(2) cells. Figure presents JC-1 dye stained (A) NB1691 and (B) SK-N-BE(2) neuroblastoma cells that were plated following SPARC overexpression and radiation treatment and analyzed using flow cytometry (10,000 cells) at fluorescence emission wavelength of $\sim 590 \mathrm{~nm}$. (C) Quantitative analysis of flow cytometry data was graphically presented (P<0.05), Staurosporine was used as a positive control $(5 \mu \mathrm{M})$. Gene ontology analysis of (D) NB1691 and (E) SK-N-BE (2) neuroblastoma cells performed in the standard enrichment computation method to functionally profile gene sets. The gene ontology was computed using SPARC overexpressing and irradiated cells vs. untreated controls. SPARC, secreted protein acidic and rich in cysteine; $\Delta \psi$, mitochondrial membrane potential; T-control, transfection control.

enrichment of genes associated with the death receptor pathway ( $<4$ fold) and TNF receptor-associated molecules ( $<5$ fold) in NB1691 cells. In SK-N-BE(2) cells, an enrichment of genes associated with HSP90 binding ( $<4$ fold) and calcium channel regulator activity ( $<4$ fold) were observed (Fig. 4 D and E).

Overexpression of SPARC accompanied with irradiation induces activation of caspase 3/7. Caspase activation studies demonstrated that SPARC overexpression increased apoptotic events by $16 \pm 4 \%$ in NB1691 cells and by $24 \pm 8 \%$ in SK-N-BE(2) neuroblastoma cells, and the addition of irradiation increased the number of apoptotic events by $60 \pm 5 \%$ in NB1691 when compared to untreated controls $(\mathrm{P}<0.05)$ and by $67 \pm 12 \%$ in SK-N-BE(2) cells compared with untreated controls $(\mathrm{P}<0.05$; Fig. 5A and B).

\section{Discussion}

SPARC, which is also known as osteonectin, belongs to the matricellular family of secreted proteins $(24,25)$. A number of biological functions in human cancer have been reported to involve SPARC. In neuroblastoma, SPARC expression has been found to be associated with impaired tumor growth and angiogenesis $(26,27)$. A previous study demonstrated that the overexpression of SPARC induced autophagy-mediated apoptosis in primitive neuroectodermal tumor cells (13). The present study investigates the underlying mechanisms prior to the induction of apoptosis. SK-N-BE2 and NB1691 neuroblastoma cell lines were subjected to overexpression of SPARC with and without ionizing radiation treatment. To determine the potential for resistance to irradiation, the cells were screened for established radiation response molecules and it was observed that HSP27 was overexpressed in NB1691 and SK-N-BE(2) cells following radiation treatment. HSP27, also known as heat shock protein beta-1, is an inhibitor of apoptosis $(28,29)$. Previous studies have reported that HSP27 protects against caspase-3 activation thereby inhibiting caspase-3 mediated apoptosis (30-32). Previous studies have also demonstrated that the overexpression of HSP27 reduces the levels of apoptosis (33) and inhibition of HSP27 radio-sensitizes tumor cells (34). Notably, a previous study suggested that HSP27-mediates gemcitabine sensitivity in pancreatic cancer cells indicating a pro-apoptotic role of HSP27 as well as the extrinsic apoptosis pathway function (35). Another study has identified that the downregulation of HSP27 increases the sensitivity of pancreatic cancer cells to gemcitabine (36). These contradictory results demonstrate that tumors cells differ and targeting tumors based on one single observation may not be clinically relevant. In the present study, two cell lines with varied p53 backgrounds were used. NB1691 is wild type for p53, and SK-N-BE(2) has a p53 mutation, and using these cell lines, it was observed that the effects of SPARC overexpression were not contradictory with 

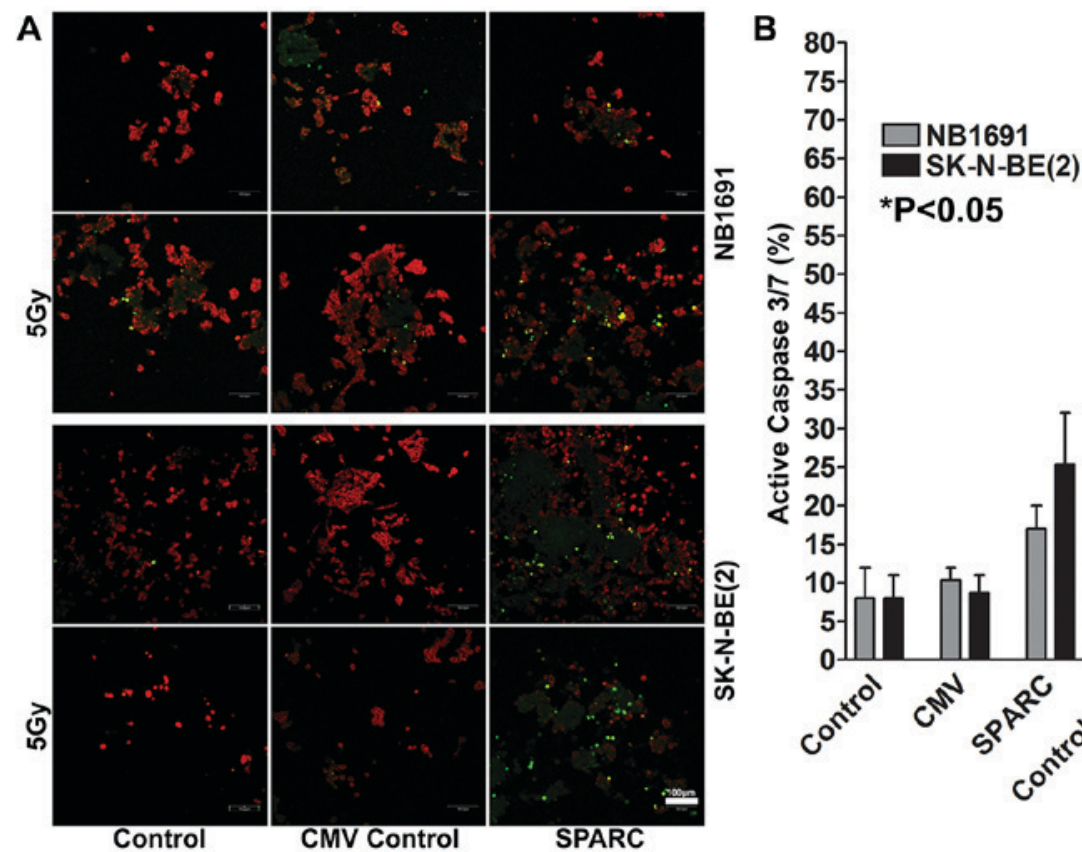

${ }^{*} \mathrm{P}<0.05$

Figure 5. Overexpression of SPARC and radiation treatment induces activation of caspase 3/7 in in NB1691 and SK-N-BE(2) cells. Figure presents caspase activation using the CellEvent Caspase3/7 Green Ready Probes from Molecular Probes. SK-N-BE(2) and NB1691 cells were plated in chamber slides and transfected with SPARC overexpression plasmid and treated with 5 Gy of ionizing radiation. CellEvent Caspase3/7 Green Ready Probe stain was performed and (A) cells were observed using a confocal microscope with green florescence indicating caspase 3/7 activation; propidium iodide was used as a counter stain to visualize non-apoptotic cells seen as red. Nuclei were visualized using propidium iodide (Scale bar, $100 \mu \mathrm{m}$ ). (B) The percentage of cells with activated caspase $3 / 7$ was determined by counting the number of cells with green florescence in 10 random fields. SPARC, secreted protein acidic and rich in cysteine; CMV, CMV, cells transfected with control plasmid containing CMV promoter with no SPARC insert.

previous studies where the status of p53 or other mutations were not indicated $(35,36)$. Studies that were published within the previous decade, reported that HSP27 may serve a role in the regulation of cellular senescence via the modulation of the p53 pathway, in which HSP27 downregulation was associated with the stabilization of p53 suggesting that HSP27 expression may suppresses p53 function (37). Although the current study observed that radiation-induced expression of HSP27 did not promote the inhibition of apoptosis as demonstrated previously (38). This indicates that radiation accompanied by SPARC overexpression may be a viable therapeutic option for neuroblastoma. Notably, the results of the current study also identified that SPARC induces the reduction of the mitochondrial $\Delta \psi$ and activates caspases, and radiation exposure alone did not significantly influence mitochondrial $\Delta \psi$. This suggests that SPARC may activate the mitochondrial-associated cell death pathway and promote the radiation-induced interjection of HSP27 that reverses apoptotic induction. However, as SPARC overexpression suppresses radiation-induced HSP27 and also activates caspases, this may suppress any rescue of SPARC-induced mitochondrial $\Delta \psi$ collapse-mediated cell death $(39,40)$.

In conclusion, the results of the present study demonstrate that SPARC overexpression suppresses radiation-induced G2M arrest and HSP27, independently of p53 status in human neuroblastoma cells.

\section{Acknowledgements}

The present study was supported by the National Cancer Institute (Rockville, MD, USA; grant no. R01CA147792) and the Department of Internal Medicine University of Illinois
College of Medicine, Peoria, IL 61605, USA. Services provided by Arraystar (Arraystar, Inc., Rockville, MD, USA) for micro array analysis and gene ontology analysis is acknowledged.

\section{References}

1. Louis CU and Shohet JM: Neuroblastoma: Molecular pathogenesis and therapy. Annu Rev Med 66: 49-63, 2015.

2. Pinto NR, Applebaum MA, Volchenboum SL, Matthay KK, London WB, Ambros PF, Nakagawara A, Berthold F, Schleiermacher G, Park JR, et al: Advances in risk classification and treatment strategies for neuroblastoma. J Clin Oncol 33: 3008-3017, 2015.

3. PDQ Pediatric Treatment Editorial Board: Neuroblastoma treatment $(\mathrm{PDQ}(\mathrm{R}))$ : Health professional version. In: PDQ cancer information summaries, Bethesda (MD), 2002.

4. Luksch R, Castellani MR, Collini P, De Bernardi B, Conte M, Gambini C, Gandola L, Garaventa A, Biasoni D, Podda M, et al: Neuroblastoma (peripheral neuroblastic tumours). Crit Rev Oncol Hematol 107: 163-181, 2016.

5. Huynh MH, Sage EH and Ringuette M: A calcium-binding motif in SPARC/osteonectin inhibits chordomesoderm cell migration during xenopus laevis gastrulation: Evidence of counter-adhesive activity in vivo. Dev Growth Differ 41: 407-418, 1999.

6. Clark CJ and Sage EH: A prototypic matricellular protein in the tumor microenvironment-where there's SPARC, there's fire. J Cell Biochem 104: 721-732, 2008.

7. Nagaraju GP and Sharma D: Anti-cancer role of SPARC, an inhibitor of adipogenesis. Cancer Treat Rev 37: 559-566, 2011.

8. Han W, Cao F, Chen MB, Lu RZ, Wang HB, Yu M, Shi CT and Ding HZ: Prognostic value of SPARC in patients with pancreatic cancer: A systematic review and meta-analysis. PLoS One 11: $\mathrm{e} 0145803,2016$.

9. Feng $\mathbf{J}$ and Tang L: SPARC in tumor pathophysiology and as a potential therapeutic target. Curr Pharm Des 20: 6182-6190, 2014.

10. Said N and Motamed K: Absence of host-secreted protein acidic and rich in cysteine (SPARC) augments peritoneal ovarian carcinomatosis. Am J Pathol 167: 1739-1752, 2005. 
11. Tang MJ and Tai IT: A novel interaction between procaspase 8 and SPARC enhances apoptosis and potentiates chemotherapy sensitivity in colorectal cancers. J Biol Chem 282: 34457-34467, 2007.

12. Chlenski A, Guerrero LJ, Peddinti R, Spitz JA, Leonhardt PT, Yang Q, Tian Y, Salwen HR and Cohn SL: Anti-angiogenic SPARC peptides inhibit progression of neuroblastoma tumors. Mol Cancer 9: 138, 2010.

13. Bhoopathi P, Chetty C, Gujrati M, Dinh DH, Rao JS and Lakka S: Cathepsin B facilitates autophagy mediated apoptosis in SPARC overexpressed primitive neuroectodermal tumor cells. Cell Death Differ 17: 1529-1539, 2010

14. Bhoopathi P, Gorantla B, Sailaja GS, Gondi CS, Gujrati M, Klopfenstein JD and Rao JS: SPARC overexpression inhibits cell proliferation in neuroblastoma and is partly mediated by tumor suppressor protein PTEN and AKT. PLoS One 7: e36093, 2012

15. Golembieski WA, Thomas SL, Schultz CR, Yunker CK, McClung HM, Lemke N, Cazacu S, Barker T, Sage EH, Brodie C and Rempel SA: HSP27 mediates SPARC-induced changes in glioma morphology, migration, and invasion. Glia 56: 1061-1075, 2008.

16. Blattes GB, Mestieri LB, Böttcher DE, Fossati AC, Montagner F and Grecca FS: Cell migration, viability and tissue reaction of calcium hypochlorite based-solutions irrigants: An in vitro and in vivo study. Arch Oral Biol 73: 34-39, 2017.

17. Grada A, Otero-Vinas M,Prieto-Castrillo F, Obagi Z and Falanga V: Research techniques made simple: Analysis of collective cell migration using the wound healing assay. J Invest Dermatol 137: e11-e16, 2017.

18. Salvioli S, Dobrucki J, Moretti L, Troiano L, Fernandez MG Pinti M, Pedrazzi J, Franceschi C and Cossarizza A: Mitochondrial heterogeneity during staurosporine-induced apoptosis in HL60 cells: Analysis at the single cell and single organelle level. Cytometry 40 : 189-197, 2000.

19. Wang Y, Perchellet EM, Ward MM, Lou K, Hua DH and Perchellet JP: Rapid collapse of mitochondrial transmembrane potential in HL-60 cells and isolated mitochondria treated with anti-tumor 1,4-anthracenediones. Anticancer Drugs 16: 953-967, 2005.

20. Hawley-Nelson P and Ciccarone V: Transfection of cultured eukaryotic cells using cationic lipid reagents. Curr Protoc Neurosci Appendix 1: Appendix 1F, 2001.

21. Paul CA, Beltz B and Berger-Sweeney J: Subbing slides. CSH Protoc 2008: pdb prot4804, 2008

22. Chadderton T, Wilson C, Bewick M and Glück S: Evaluation of three rapid RNA extraction reagents: Relevance for use in RT-PCR's and measurement of low level gene expression in clinical samples. Cell Mol Biol (Noisy-le-Grand) 43: 1227-1234, 1997.

23. Mi H, Muruganujan A, Casagrande JT and Thomas PD: Large-scale gene function analysis with the PANTHER classification system. Nat Protoc 8: 1551-1566, 2013.

24. Lane TF and Sage EH: The biology of SPARC, a protein that modulates cell-matrix interactions. FASEB J 8: 163-173, 1994.

25. Porter PL, Sage EH, Lane TF, Funk SE and Gown AM: Distribution of SPARC in normal and neoplastic human tissue. J Histochem Cytochem 43: 791-800, 1995.
26. Gorantla B, Bhoopathi P, Chetty C, Gogineni VR, Sailaja GS, Gondi CS and Rao JS: Notch signaling regulates tumor-induced angiogenesis in SPARC-overexpressed neuroblastoma. Angiogenesis 16: 85-100, 2013.

27. Ribatti D: Anti-angiogenesis in neuroblastoma. Crit Rev Oncol Hematol 86: 212-221, 2013.

28. Arya R, Mallik M and Lakhotia SC: Heat shock genes-integrating cell survival and death. J Biosci 32: 595-610, 2007.

29. Bao XQ and Liu GT: Bicyclol protects HepG2 cells against D-galactosamine-induced apoptosis through inducing heat shock protein 27 and mitochondria associated pathway. Acta Pharmacol Sin 31: 219-226, 2010.

30. Pandey P, Farber R, Nakazawa A, Kumar S, Bharti A, Nalin C, Weichselbaum R, Kufe D and Kharbanda S: Hsp27 functions as a negative regulator of cytochrome c-dependent activation of procaspase-3. Oncogene 19: 1975-1981, 2000.

31. Li R, Li J, Sang D and Lan Q: Phosphorylation of AKT induced by phosphorylated Hsp27 confers the apoptosis-resistance in t-AUCB-treated glioblastoma cells in vitro. J Neurooncol 121: 83-89, 2015.

32. Hsu HS, Lin JH, Huang WC, Hsu TW, Su K, Chiou SH, Tsai YT and Hung SC: Chemoresistance of lung cancer stemlike cells depends on activation of Hsp27. Cancer 117: 1516-1528, 2011.

33. Zhang B, Zhou HS, Cheng Q, Lei L and Hu B: Overexpression of HSP27 in cultured human aortic smooth muscular cells reduces apoptosis induced by low-frequency and low-energy ultrasound by inhibition of an intrinsic pathway. Genet Mol Res 12: 6588-6601, 2013.

34. Guttmann DM, Hart L, Du K, Seletsky A and Koumenis C: Inhibition of Hsp27 radiosensitizes head-and-neck cancer by modulating deoxyribonucleic acid repair. Int J Radiat Oncol Biol Phys 87: 168-175, 2013.

35. Guo Y, Ziesch A, Hocke S, Kampmann E, Ochs S, De Toni EN, Göke B and Gallmeier E: Overexpression of heat shock protein 27 (HSP27) increases gemcitabine sensitivity in pancreatic cancer cells through S-phase arrest and apoptosis. J Cell Mol Med 19: 340-350, 2015.

36. Zhang S, Zhang XQ, Huang SL, Chen M, Shen SS, Ding XW, Lv Y and Zou XP: The effects of HSP27 on gemcitabine-resistant pancreatic cancer cell line through snail. Pancreas 44 1121-1129, 2015.

37. O'Callaghan-Sunol C, Gabai VL and Sherman MY: Hsp27 modulates p53 signaling and suppresses cellular senescence. Cancer Res 67: 11779-11788, 2007.

38. Sailaja GS, Bhoopathi P, Gorantla B, Chetty C, Gogineni VR, Velpula KK, Gondi CS and Rao JS: The secreted protein acidic and rich in cysteine (SPARC) induces endoplasmic reticulum stress leading to autophagy-mediated apoptosis in neuroblastoma. Int J Oncol 42: 188-196, 2013.

39. Fulda S: Targeting apoptosis for anticancer therapy. Semin Cancer Biol 31: 84-88, 2015.

40. Kim B, Srivastava SK and Kim SH: Caspase-9 as a therapeutic target for treating cancer. Expert Opin Ther Targets 19: $113-127,2015$. 